\title{
Curcumin suppresses migration and proliferation of Hep3B hepatocarcinoma cells through inhibition of the Wnt signaling pathway
}

\author{
HYUN JUNG KIM ${ }^{1}$, SONG YI PARK ${ }^{1}$, OCK JIN PARK ${ }^{2}$ and YOUNG-MIN KIM ${ }^{1}$ \\ Departments of ${ }^{1}$ Biological Sciences and Biotechnology and ${ }^{2}$ Food and Nutrition, \\ Hannam University Daedeok Valley Campus, Daejeon 305-811, Republic of Korea
}

Received January 15, 2013; Accepted April 29, 2013

DOI: $10.3892 / \mathrm{mmr} .2013 .1497$

\begin{abstract}
Curcumin, the major phytochemical in turmeric, exerts anti-proliferative, anticancer and anti-inflammatory activities in various types of cancer cells. Curcumin has been demonstrated to induce apoptosis through multiple signaling pathways; however, its association with survival pathways, including the Wnt signaling pathway, is not fully understood. The Wnt signaling pathway is involved in diverse functions, including cell development, growth and proliferation. This pathway is important for cancer cell survival and metastasis. $\beta$-catenin and GSK3 $\beta$ play a key role in the Wnt signaling pathway and therefore, various members of the Wnt signaling pathway have been hypothesized to represent potential targets for anticancer therapy. In the present study, the effect of curcumin on the suppression of migration and proliferation of Hep3B hepatocarcinoma cells was investigated via suppression of Wnt signaling in vitro and in vivo. 12-O-tetradecanoylphorbol-13-acetate (TPA)-induced cell migration was observed to be suppressed by curcumin treatment. In addition, curcumin suppressed TPA-induced activation of Wnt signaling. These results indicate that curcumin induces anti-migratory activity, which functions via the Wnt signaling pathway.
\end{abstract}

\section{Introduction}

Curcumin, the major phytochemical in turmeric, has a diverse pharmacological role, including anticancer, anti-inflammatory, antioxidant and anti-proliferative activities (1). In a previous study, curcumin was identified to exhibit anticancer

Correspondence to: Professor Young-Min Kim, Department of Biological Sciences and Biotechnology, Hannam University Daedeok Valley Campus, 461-6 Jeonmin-dong, Yuseong-gu, Daejeon 305-811, Republic of Korea

E-mail: kym@hnu.kr

Key words: curcumin, apoptosis, migration, Wnt signaling pathway, hepatocarcinoma effects by interfering with signaling pathways associated with the initiation, promotion and progression of multistage carcinogenesis (2). In addition, curcumin has been found to induce apoptosis in various types of cancer cells, including colon, breast, lung and ovarian (3-6), and suppresses tumor growth in various cancer xenograft models $(7,8)$. Curcumin inhibits metastasis and angiogenesis by suppression of matrix metalloproteinase-9, vascular endothelial growth factor and hypoxia-inducible factor $1 \alpha$ expression in hepatocarcinoma (9).

The Wnt signaling pathway is important in cell growth, proliferation, differentiation and development. Several studies have reported that overactivation of $\beta$-catenin in the cytosol is associated with cancer metastasis (10-13). Under normal conditions, $\beta$-catenin is phosphorylated at the Ser33/37 residue by GSK3 $\beta$ in the GSK3 $\beta / \mathrm{Axin} / \mathrm{Ck} 1$ complex, triggering subsequent proteasomal degradation. Phosphorylated $\beta$-catenin translocates into the nucleus, where it activates target genes coding for proteins, including cyclin D and c-Myc in the Wnt pathway, by binding with the transcription factor, T cell factor (TCF) (14). The Akt/mTOR pathway, in addition to overactivated Wnt signaling, inhibits GSK $3 \beta$ activity. Phosphorylation of GSK3 $\beta$ by Akt has been demonstrated in a number of cell lines to promote angiogenesis, metastasis and cell survival by activation of the $N F-\kappa B$ signaling pathway (15-18).

Although several anticancer effects of curcumin in cancer cells have been reported, studies on the molecular mechanisms of these effects via the survival pathways in hepatocarcinoma have not been performed. Curcumin possesses the ability to suppress Wnt signaling and may be important for the development of anti-proliferative and anti-metastatic drugs.

In the current study, curcumin was observed to induce apoptosis by cell proliferation assay and cell cycle analysis. Curcumin induced expression of apoptotic proteins and suppressed Wnt signaling by the reduction of $\beta$-catenin and phospho-GSK $3 \beta$ in vitro. In addition, in vivo suppression of the Wnt signaling pathway by curcumin was identified. Curcumin significantly suppressed $12-O$-tetradecanoylphorbol-13-acetate (TPA)-induced cell migration by blocking the Wnt signaling pathway in Hep3B hepatocarcinoma cells. 


\section{Materials and methods}

Cell culture and reagents. Hep3B cells were obtained from the American Type Culture Collection (Manassas, VA, USA). Cells were grown in DMEM containing $10 \%$ fetal bovine serum and $1 \%$ antibiotics at $37^{\circ} \mathrm{C}$ in a $5 \% \mathrm{CO}_{2}$ incubator. Curcumin, TPA, 3-(4,5-dimethylthiazol-2-yl)-2,5-diphenyltetrazolium bromide (MTT), propidium iodide (PI) and BIO were obtained from Sigma-Aldrich (St. Louis, MO, USA). LY294002 was purchased from Tocris Bioscience (Bristol, UK).

MTT assay. Cells seeded in 12-well plates at $1 \times 10^{5}$ cells $/ \mathrm{ml}$ were incubated with curcumin for indicated times and concentrations. Respective medium was removed and then incubated with $20 \mu \mathrm{l} \mathrm{MTT} \mathrm{solution} \mathrm{(5} \mathrm{mg/ml} \mathrm{MTT} \mathrm{in} \mathrm{PBS)} \mathrm{for} 1 \mathrm{~h}$. Converted purple formazan dye from MTT was solubilized in DMSO and optical densities were measured at $595 \mathrm{~nm}$.

Cell cycle analysis. Cells treated with curcumin at various concentrations $(10,20$ and $40 \mu \mathrm{g} / \mathrm{ml}$ for $24 \mathrm{~h})$ were harvested, washed with phosphate-buffered saline (PBS) and fixed in $70 \%$ cold ethanol. Following washing with PBS, cells were resuspended in PBS and incubated with RNase A and PI. Cells were analyzed with flow cytometry.

Wound healing assay. Hep3B cells were grown to $90 \%$ confluence in a 6 -well plate at $37^{\circ} \mathrm{C}$ in a $5 \% \mathrm{CO}_{2}$ incubator. A wound was created by scratching cells with a sterile $200 \mu$ l pipette tip. Cells were washed with PBS to remove floating cells and then added to a medium. The distance between wound edges was measured at a fixed region. Images of the wound were captured under a microscope (magnification, x100).

Western blot analysis. Cells were washed with PBS and lysed with RIPA lysis buffer [50 mM Tris- $\mathrm{HCl}$ (pH 8.0), $1 \%$ NP-40, $0.5 \%$ sodium deoxycholate, $150 \mathrm{mM} \mathrm{NaCl}$ and $1 \mathrm{mM}$ PMSF] Protein concentrations were determined using the Bradford assay. All samples were separated by sodium dodecyl sulfate polyacrylamide gel electrophoresis and transferred onto nitrocellulose membrane. The membrane was incubated overnight with primary antibodies. Secondary antibodies, conjugated to horseradish peroxidase, against IgG were used. Proteins were visualized by enhanced chemiluminescence (Intron Biotechnology, Seong nam, Korea) and detected using the LAS4000 chemiluminescence detection system (Fujifilm, Tokyo, Japan).

Xenograft and immunohistochemistry. Hep3B cells $\left(2 \times 10^{6}\right)$ were inoculated subcutaneously in 4-week nu/nu mice at the left flank. After 1 week, mice were administered curcumin or PBS (control) by injection. Tumor size was measured in two perpendicular diameters by using a caliper every 3 days and tumor volume was calculated using the following formula: Volume $=1 / 2 \times\left(\right.$ length $\mathrm{x}$ width $\left.{ }^{2}\right)$.

Tumor specimens from mice were fixed in $10 \%$ formaldehyde, embedded in paraffin and sectioned into $5-\mu \mathrm{m}$ thick slices. Sections were deparaffinized with xylene and dehydrated with $98 \%$ ethanol. Serial sections were stained using standard immunoperoxidase techniques with primary antibodies against CD31. For epitope retrieval, specimens were microwave treated for $25 \mathrm{~min}$ prior to incubation with primary antibody. Pre-immune serum was used as a negative control for immunostaining and positive staining was visualized with diaminobenzidine, followed by a light counterstaining with hematoxylin. Sections were evaluated by a pathologist, determining stain intensity and percentage of reactive cells. Representative images were captured. All animal experiments were approved by the Ethics Committee for Animal Experimentation of the Hannam University (Daejeon, South Korea).

Statistical analysis. Cell viability data were statistically analyzed using unpaired t-tests (SPSS, Inc., Chicago, IL, USA). $\mathrm{P}<0.05$ was considered to indicate a statistically significant difference.

\section{Results}

Effect of curcumin on cell viability and apoptosis in Hep3B cells. To examine the effects of curcumin on Hep3B cell proliferation, cells were seeded in 12-well plates at a density of $1 \times 10^{5}$ cells/well. The effect of curcumin on cell viability was determined by MTT assay. Fig. 1A demonstrates significant inhibition of cell proliferation in curcumin-treated cells in a dose- and time-dependent manner, compared with control cells. Cell cycle analysis revealed that curcumin induced dose-dependent apoptosis. The percentage of apoptotic cells (sub- $\mathrm{G}_{1}$ peak) increased from $19.5 \%$ (control) to $35.53 \%$ (curcumin $20 \mu \mathrm{M}$ ) and $32.15 \%$ (curcumin $40 \mu \mathrm{M}$ ) in the Hep3B hepatocarcinoma cells (Fig. 1B). To determine the mechanism of curcumin-induced apoptosis, the expression levels of apoptotic proteins, including procaspase-3, PARP, Bax and Bcl-2, were detected by western blot analysis. Activation of caspase-3 plays a critical role in apoptosis, as it proteolytically cleaves PARP. In Hep3B cells treated with curcumin, the levels of procaspase-3, PARP and Bcl-2 decreased and Bax increased.

Effect of curcumin on the Wnt signaling pathway in vitro and in vivo. The Wnt signaling pathway plays a critical role in tumor cell growth and survival in hepatocarcinoma. Therefore, the inhibitory effect of curcumin on Wnt signaling in Hep3B cells was determined by analyzing the levels of $\beta$-catenin and GSK $3 \beta$, which play key roles in the pathway. Previous studies have demonstrated that GSK3 $\beta$ is inactivated by Akt and regulated by mTOR (25). In addition, Akt and mTOR are associated with cell survival and growth. Therefore, levels of $\beta$-catenin, p-GSK3 $\beta$, p-Akt and p-mTOR were assessed by western blot analysis. As demonstrated in Fig. 2A, curcumin increased phosphorylation of GSK $3 \beta$, Akt and mTOR and thus, the level of $\beta$-catenin, in a dose-dependent manner. To explore the therapeutic effects of curcumin, hepatocarcinoma tumors were established in nude mice and tumors were treated by injecting curcumin. As revealed in Fig. 2B, tumor volume of the curcumin-treated group was significantly reduced compared with that of the control group. No significant differences in the body weight of mice were observed between control and curcumin-treated groups. Furthermore, curcumin was observed to induce antitumor activity by suppression of Wnt signaling molecules. Fig. 2C demonstrates that $\beta$-catenin, p-mTOR, p-Akt and p-GSK3 $\beta$ expression was significantly 

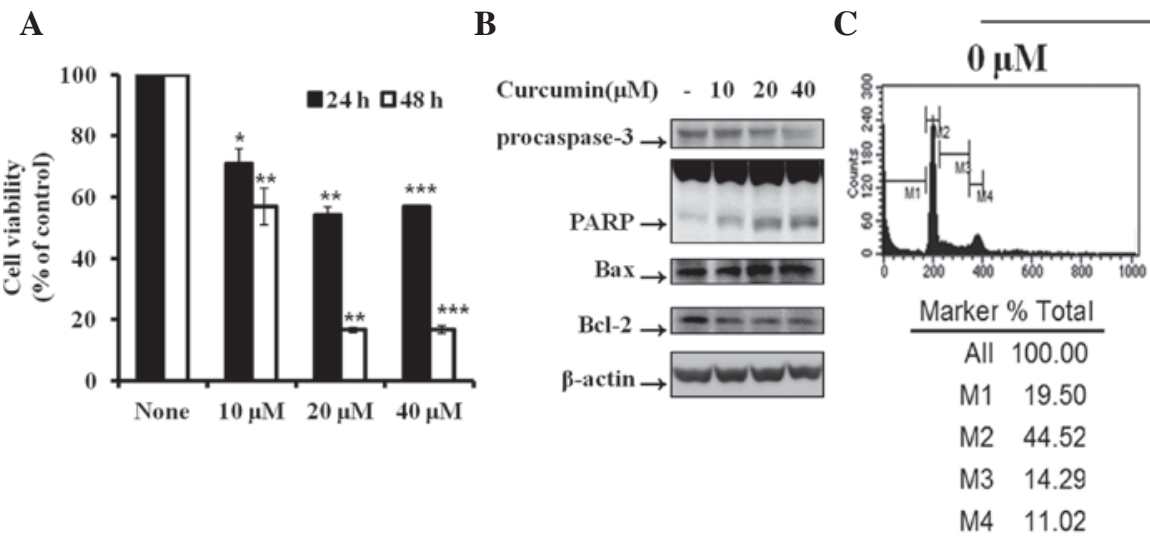

Curcumin, 24 h

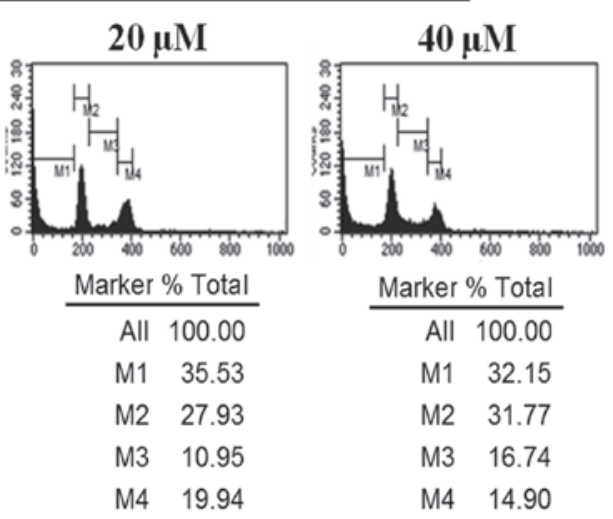

Figure 1. Cytotoxicity and cell apoptotic effects in Hep3B cells induced by curcumin. (A) Hep3B cells were treated with various concentrations of curcumin for various times and cell viability was detected by MTT assay. Data are presented as the mean \pm SD. (B) Protein expression of procaspase-3, PARP, Bax and $\mathrm{Bcl}-2$ in Hep3B cells treated with various concentrations of curcumin for $6 \mathrm{~h}$ was determined. (C) Hep3B cells were treated with various concentrations of curcumin for $24 \mathrm{~h}$ and the cell cycle percentage was determined by FACS. M1, sub- $\mathrm{G}_{1}$ phase; $\mathrm{M} 2, \mathrm{G}_{0} / \mathrm{G}_{1}$ phase; M3, S phase; M4, G $/ \mathrm{M}$ phase; FACS, fluorescence-activated cell sorting; MTT, 3-(4,5-dimethylthiazol-2-yl)-2,5-diphenyltetrazolium bromide.

A

C

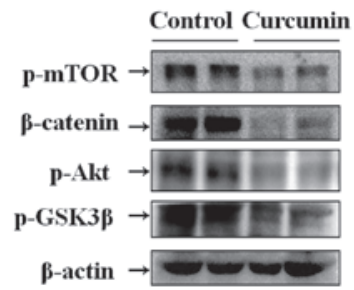

B

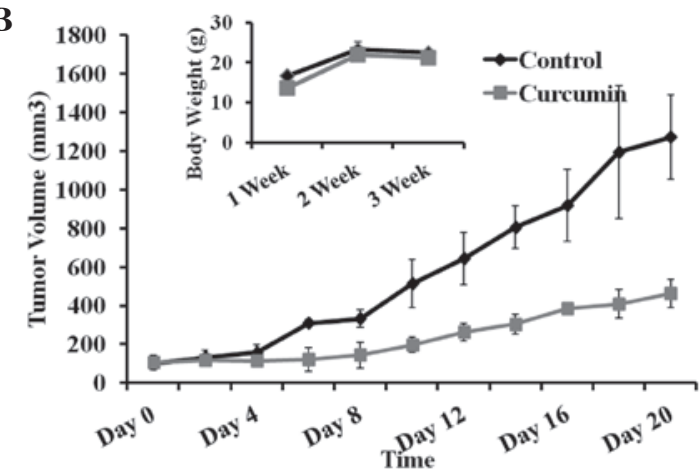

D

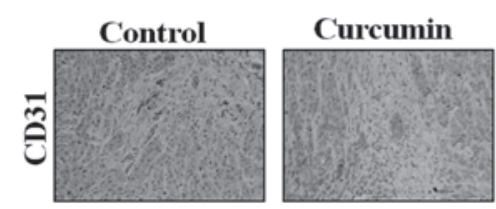

Figure 2. Curcumin suppressed p-mTOR, p-Akt, $\beta$-catenin and p-GSK3 $\beta$ expression in vitro and in vivo. (A) When the concentration of curcumin increased, the expression of p-mTOR, p-Akt, $\beta$-catenin and p-GSK3 $\beta$ was significantly decreased in Hep3B cells. (B) Curcumin-treated tumors exhibited significantly reduced growth compared with control. (C) Tumor lysates were analyzed by western blot analysis. (D) Tumor tissue sections were analyzed by immunohistochemistry using anti-CD31 antibody for the detection of microvessel density.

decreased in the curcumin-treated group compared with the control. To further correlate these observations, in vivo tumor therapeutic effects were assessed in vitro. Thus, the expression levels of biomarkers were assessed in tumor tissues by immunohistochemical analysis. The results indicated that CD31-positive microvessels were significantly suppressed following curcumin treatment (Fig. 2D).

Effect of curcumin on TPA-induced Wnt signaling pathway activation and cell migration in Hep $3 B$ cells. To investigate the effect of curcumin on TPA-induced Wnt signaling activation and cell migration, wound healing assays and western blot analysis were performed in Hep3B cells. Western blot analysis demonstrated that TPA increased $\beta$-catenin and phos- phorylation of mTOR, Akt and GSK3 $\beta$, and that curcumin inhibited TPA-induced Wnt signaling pathway activation at $6 \mathrm{~h}$ in a concentration-dependent manner (Fig. 3A and B). As demonstrated in Fig. 3C, cell migration was increased in cells treated with TPA. However, the number of migrated cells was significantly decreased in the group treated with curcumin alone and the group pre-treated with TPA and then followed with curcumin for $48 \mathrm{~h}$ (for 12 and $24 \mathrm{~h}$; data not shown).

Effect of curcumin with LY294002 or BIO on Wnt signaling. Western blot analysis was used to clarify the mechanism of curcumin-mediated suppression of the Wnt signaling pathway in Hep3B cells by the suppression of specific molecules. The results indicate that curcumin significantly suppressed 

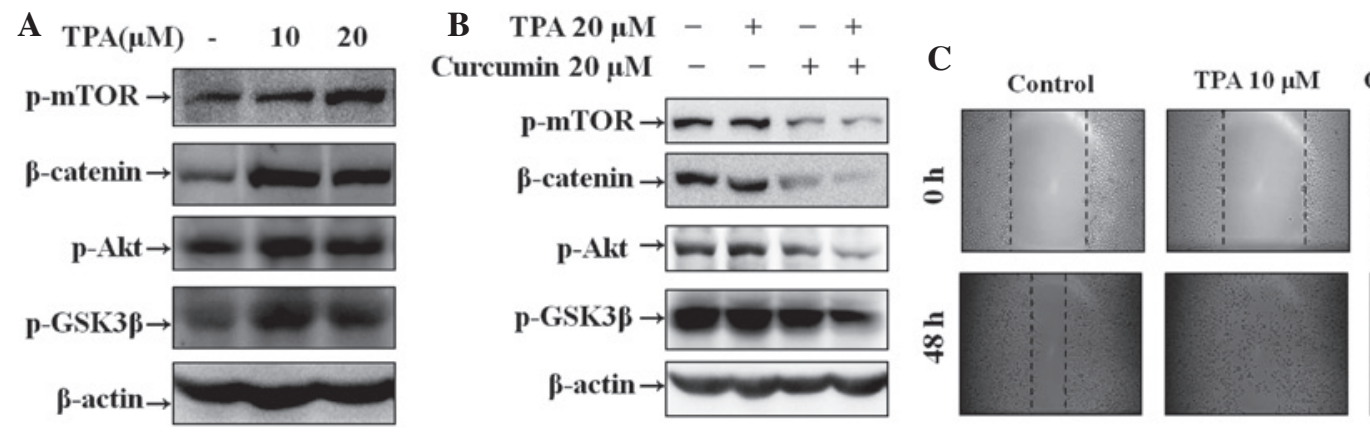

TPA $10 \mu \mathrm{M}+$

Figure 3. Effect of curcumin on TPA-induced p-mTOR, p-Akt, $\beta$-catenin and p-GSK3 $\beta$ expression, and cell migration in Hep3B cells. Cells were treated with TPA or curcumin alone, and pretreated with TPA for $30 \mathrm{~min}$, followed by treatment with curcumin. (A and B) Protein levels of p-mTOR, p-Akt, $\beta$-catenin and p-GSK3 $\beta$ were detected by western blot analysis. (C) Cell migration in Hep3B cells was detected by wound healing assay. TPA, 12- $O$-tetradecanoylphorbol-13-acetate.

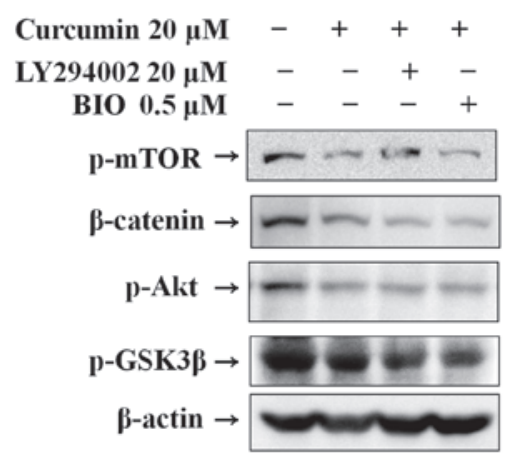

Figure 4. Effect of curcumin with LY294002 or BIO on p-mTOR, p-Akt, $\beta$-catenin and $p$-GSK3 $\beta$ expression in Hep3B cells. Hep3B cells were pretreated with LY294002 or BIO for $30 \mathrm{~min}$, followed with curcumin for $6 \mathrm{~h}$.

the expression of p-mTOR, p-Akt, $\beta$-catenin and p-GSK3 $\beta$. Pre-treatment using LY294002 and BIO, specific PI3K and GSK3 inhibitors, respectively, followed by curcumin treatment, effectively reduced $\beta$-catenin and phosphorylation of mTOR, Akt and GSK3 $\beta$ (Fig. 4).

\section{Discussion}

Liver cancer is the leading cause of mortality from cancer and is more prevalent in Asia and Africa. To date, a number of signaling pathways, including Ras/Raf/MEK/ERK, $\mathrm{PI} 3 \mathrm{~K} / \mathrm{Akt} / \mathrm{mTOR}$ and $\mathrm{NF}-\kappa \mathrm{B}$ have been hypothesized to represent potential targets for hepatocarcinoma therapy. Thus, the Wnt signaling pathway is markedly involved in the development of normal cells as well as tumorigenesis. In addition, mutation and aberrant activation of the Wnt signaling pathway has been identified in various types of cancer and is frequently activated in hepatocarcinoma (19). Activation of Wnt signaling results in phosphorylation of GSK3 $\beta$ (inactive form), which in turn leads to transcription of oncogenes by $\beta$-catenin translocation and binding with TCF in the nucleus. Following this, the complex activates the transcription of growth-promoting genes, including cyclin D and c-Myc. Previous studies have reported that curcumin represents a potent anticancer agent. In addition, studies have demonstrated that Bcl-2, Bax, procaspase-3, PARP, Noxa and PUMA may serve as predictive markers for the evaluation of apoptosis in various cancer cells $(20,21)$. Thus, in the present study, curcumin was hypothesized to suppress cell migration and proliferation by inhibiting Wnt signaling. Curcumin was found to suppress cell proliferation in vitro by blocking Wnt signaling and inhibition of the pathway, which correlated with the suppression of cell migration. In addition, analysis of the cell cycle and expression of apoptotic proteins was performed, confirming that curcumin induced apoptosis. Curcumin was also identified to suppress the expression of $\mathrm{p}$-mTOR, $\mathrm{p}$-Akt, $\beta$-catenin and p-GSK3 $\beta$ regardless of treatment with inhibitors (LY294002 and $\mathrm{BIO})$.

TPA, a tumor-promoting phorbol ester, enhances cellular signaling pathways, including PI3K/Akt, PKC and MAPK (22). TPA also activates the PI3K pathway and downstream Akt, leading to inhibition of GSK3 $\beta$. In addition, TPA-induced isoforms of PKC has been observed to inactivate GSK3 $\beta$. This inactivation leads to $\beta$-catenin accumulation and Wnt target gene expression, which are involved in cell proliferation $(23,24)$. In the present study, migration triggered by TPA was found to be suppressed by curcumin, confirming that curcumin inhibits TPA-induced Wnt signaling activation. In addition, curcumin was observed to suppress tumor growth and microvessel density by blocking the Wnt signaling pathway in vivo.

In conclusion, results of the present study indicate that curcumin induces apoptosis, as confirmed by cell proliferation assays, analysis of the cell cycle and expression of apoptotic proteins. Curcumin was also found to inhibit TPA-induced migration in vitro as well as tumor growth in vivo through inhibition of the Wnt signaling pathway. Thus, curcumin treatment may be developed as a novel strategy for the suppression of cell proliferation and survival in hepatocarcinoma.

\section{Acknowledgements}

The present study was supported by a grant from the National Research Foundation of Korea (KRF-2012-0021402).

\section{References}

1. Surh YJ: Cancer chemoprevention with dietary phytochemicals. Nat Rev Cancer 3: 768-780, 2003.

2. Thangapazham RL, Sharma A and Maheshwari RK: Multiple molecular targets in cancer chemoprevention by curcumin. AAPS J 8: 443-449, 2006. 
3. Johnson JJ and Mukhtar H: Curcumin for chemoprevention of colon cancer. Cancer Lett 255: 170-181, 2007.

4. Radhakrishna Pillai G, Srivastava AS, Hassanein TI, Chauhan DP and Carrier E: Induction of apoptosis in human lung cancer cells by curcumin. Cancer Lett 208: 163-170, 2004.

5. Shi M, Cai Q, Yao L, Mao Y, Ming Y and Ouyang G: Antiproliferation and apoptosis induced by curcumin in human ovarian cancer cells. Cell Biol Int 30: 221-226, 2006.

6. Mehta K, Pantazis P, McQueen T and Aggarwal BB Antiproliferative effect of curcumin (diferuloylmethane) agains human breast tumor cell lines. Anticancer Drugs 8: 470-481, 1997.

7. Perry MC, Demeule M, Régina A, Moumdjian R and Béliveau R: Curcumin inhibits tumor growth and angiogenesis in glioblastoma xenografts. Mol Nutr Food Res 54: 1192-1201, 2010.

8. Dujic J, Kippenberger S, Ramirez-Bosca A, Diaz-Alperi J, Bereiter-Hahn J, Kaufmann R, Bernd A and Hofmann M: Curcumin in combination with visible light inhibits tumor growth in a xenograft tumor model. Int J Cancer 124: 1422-1428, 2009.

9. Stagos D, Amoutzias GD, Matakos A, Spyrou A, Tsatsakis AM and Kouretas D: Chemoprevention of liver cancer by plant polyphenols. Food Chem Toxicol 50: 2155-2170, 2012.

10. Polakis P: Wnt signaling and cancer. Genes Dev 14: 1837-1851, 2000

11. Fatima S, Lee NP and Luk JM: Dickkopfs and Wnt/ $\beta$-catenin signalling in liver cancer. World J Clin Oncol 2: 311-325, 2011

12. Esufali S and Bapat B: Cross-talk between Rac1 GTPase and dysregulated Wnt signaling pathway leads to cellular redistribution of beta-catenin and TCF/LEF-mediated transcriptional activation. Oncogene 23: 8260-8271, 2004.

13. Whittaker S, Marais R and Zhu AX: The role of signaling pathways in the development and treatment of hepatocellular carcinoma. Oncogene 29: 4989-5005, 2010.

14. Cadigan KM and Nusse R: Wnt signaling: a common theme in animal development. Genes Dev 11: 3286-3305, 1997.

15. Kim D and Chung J: Akt: versatile mediator of cell survival and beyond. J Biochem Mol Biol 35: 106-115, 2002.

16. Nojima H, Tokunaga C, Eguchi S, Oshiro N, Hidayat S, Yoshino K, Hara K, Tanaka N, Avruch J and Yonezawa K: The mammalian target of rapamycin (mTOR) partner, raptor, binds the mTOR substrates p70 S6 kinase and 4E-BP1 through their TOR signaling (TOS) motif. J Biol Chem 278: 15461-15464, 2003.
17. Jung EM, Lim JH, Lee TJ, Park JW, Choi KS and Kwon TK: Curcumin sensitizes tumor necrosis factor-related apoptosis-inducing ligand (TRAIL)-induced apoptosis through reactive oxygen species-mediated upregulation of death receptor 5 (DR5). Carcinogenesis 26: 1905-1913, 2005.

18. Yu S, Shen G, Khor TO, Kim JH and Kong AN: Curcumin inhibits Akt/mammalian target of rapamycin signaling through protein phosphatase-dependent mechanism. Mol Cancer Ther 7: 2609-2620, 2008.

19. Jemal A, Bray F, Center MM, Ferlay J, Ward E and Forman D: Global cancer statistics. CA Cancer J Clin 61: 69-90, 2011.

20. Chiu TL and Su CC: Curcumin inhibits proliferation and migration by increasing the $\mathrm{Bax}$ to $\mathrm{Bcl}-2$ ratio and decrasing NF-Bp65 expression in breast cancer MDA-MB-231 cells. Int J Mol Med 23: 468-475, 2009.

21. Shankar S and Srivastava RK: Involvement of Bcl-2 family members, phosphatidylinositol 3'-kinase/AKT and mitochondrial p53 in curcumin (diferulolylmethane)-induced apoptosis in prostate cancer. Int J Oncol 30: 905-918, 2007.

22. Murayama K, Kimura T, Tarutani M, Tomooka M, Hayashi R, Okabe M, Nishida K, Itami S, Katayama I and Nakano T: Akt activation induces epidermal hyperplasia and proliferation of epidermal progenitors. Oncogene 26: 4882-4888, 2007.

23. Inoki $\mathrm{K}$, Ouyang $\mathrm{H}$, Zhu T, Lindvall C, Wang $\mathrm{Y}$, Zhang $\mathrm{X}$, Yang Q, Bennett C, Harada Y, Stankunas K, Wang CY, He X, MacDougald OA, You M, Williams BO and Guan KL: TSC2 integrates Wnt and energy signals via a coordinated phosphorylation by AMPK and GSK3 to regulate cell growth. Cell 126: 955-968, 2006

24. Chen RH, Ding WV and McCormick F: Wnt signaling to beta-catenin involves two interactive components. Glycogen synthase kinase-3beta inhibition and activation of protein kinase C. J Biol Chem 275: 17894-17899, 2000.

25. Dal Col J and Dolcetti R: GSK-3beta inhibition: at the crossroad between Akt and mTOR constitutive activation to enhance cyclin D1 protein stability in mantle cell lymphoma. Cell Cycle 7: 2813-2816, 2008. 